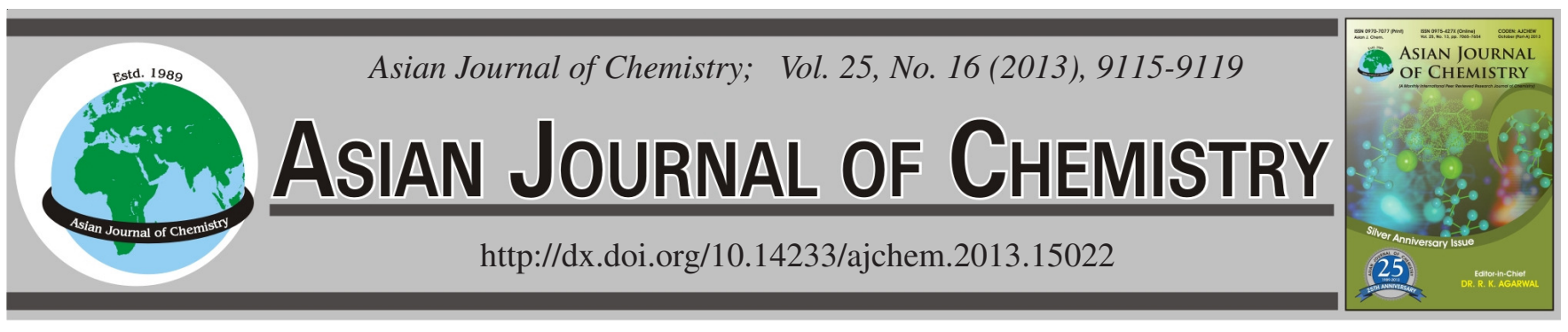

\title{
$a b$ initio Large Unit Cell Calculations of Electronic Structure of Cubic Boron Nitride Nanocrystals
}

\author{
Mudar A. Abdulsattar
}

Ministry of Science and Technology, Baghdad, Iraq

Corresponding author: E-mail: mudarahmed3@yahoo.com

\begin{abstract}
Density functional theory at the generalized gradient approximation level of Perdew-Burke-Ernzerhof coupled with the large unit cell method is used to simulate the core and surface parts of cubic boron nitride zinc blende nanocrystals. The cubic boron nitride nanocrystals in our model are represented by a heterojunction between the surface and the core in which the surface represents the outer four layers and the core by the rest of the internal region of a nanocrystal. The present model results show that boron nitride nanocrystals core has generally fluctuating energy gap as the nanocrystals grow up in size for the selected size range. The present work shows decreasing lattice constant and ionicity of the core part with increasing nanocrystal size. The lattice contraction is $2 \%$ of the size of core equilibrium lattice constant. The method also shows increasing cohesive energy (absolute value) and valence band width with increasing nanocrystal size. All the properties reach converged values nearly at 1.4-1.9 nanometer of nanocrystal size. The hydrogenated B-terminated $(001)-(1 \times 1)$ nanocrystal surface is also investigated to determine the rule of the surface in nanocrystals electronic structure. Surface results show that energy gap is controlled by the surface part since it has lower energy gap value. The highly degenerated core states are split at the surface causing the reduction of energy gap and widening of both valence and conduction bands. A comparison between the largest investigated core cells with bulk properties reflects the fast convergence of these properties to bulk values and the quality of the present method. Use of symmetry in large unit cell method leads to a huge reduction of computational time with respect to traditional full geometrical optimization.
\end{abstract}

Key Words: ab initio calculations, Cubic boron nitride, Nanocrystals, Density functional theory.

\section{INTRODUCTION}

Cubic boron nitride is a wide gap semiconductor with a variety of properties that are close to diamond. This material exists in allotropic forms such as the hexagonal form that resembles graphite ${ }^{1}$. Hardness and thermal properties of cubic boron nitride are comparable to diamond and are among the best in such properties ${ }^{2}$. Boron nitride nanotubes are also similar to carbon nanotubes in many properties ${ }^{3}$. This compound forms with carbon ternary compounds that have promising properties $^{4,5}$.

Cubic boron nitride electronic structure is one of the early theoretically and experimentally investigated structures due to its excellent mechanical and thermal properties and its resemblance with diamond structure ${ }^{2}$. Theoretical band structure of nanocrystals of boron nitride is rarely investigated with most of the investigations concentrating on the experimental part of preparation or applications $s^{6,7}$. On the other hand, diamond nanocrystals are heavily investigated both experimentally and theoretically ${ }^{8-17}$. Recent investigations showed that diamond nanocrystals energy gap beyond the quantum confinement region (exciton Bohr radius) begin to rise ${ }^{8}$. Inside the quantum confinement region the quantum confinement theory imply a decreasing value of the band gap as the nanocrystal grows up in size. In the present work we shall show that this property is not only applicable to diamond but also to cubic boron nitride.

The large unit cell method (LUC) is one of several kinds of supercell methods ${ }^{18-21}$. In this method, a central large cell is repeated periodically through out the space. To simulate or represent a single one cluster of atoms at the core of a nanocrystal, the wave vector $(\mathrm{k})$ is restricted to the value at the origin only $(\mathrm{k}=0)$ with a restricted interaction range. The LUC method is traditionally coupled to semi-empirical methods such as CNDO (Complete neglect of differentia overlap), or INDO (Intermediate neglect of differential overlap) to reduce computational time ${ }^{18-21}$. In the present work, we shall investigate the use of $a b$ initio density functional theory (DFT) combined with the LUC method (LUC-DFT) to simulate the core and surface parts of boron nitride nanocrystals. 


\section{THEORY}

In physics and chemistry of surfaces, it is customarily to take the three outermost atomic layers of a crystalline solid to represent the reconstructed surface ${ }^{22}$. In this case, the atoms of the inside fourth layer (counted from the surface) are generally at or close to their original positions as implied by the internal core lattice periodicity. According to this fact we divide nanocrystal electronic structure calculation into two parts, i.e., surface and core. The surface part is taken to be composed by the outer four layers. The fourth layer is included in the surface to ensure that first neighbors of core atoms are in their nearly exact periodical lattice positions. In very small nanocrystals the number of surface atoms is greater than core; however, the number of core atoms increases over the number of surface atoms when the nanocrystal increases in size. The present method is suitable for nanocrystals of a total of 200 atoms or greater in which a well defined regular periodic core exist with equal bond lengths and angles. The total electronic structure of the nanocrystal is the combined electronic structure of core and surface. The core part is always regular and unique in its properties for a given structure but the surface part has different reconstructions depending on kind or orientation of the surface, passivating atoms, method of formation, substrates, environment, etc. In the present work we shall investigate and compare surface and core properties of equiaxed cubic boron nitride nanocrystals.

To keep equiaxed LUCs, certain sizes of LUC have to be chosen. In the LUC method, a unit cell that is a multiple of either the Bravais or primitive cell (Fig. 1a-b) of the zinc blende structure is used to represent the core of a nanocrystal ${ }^{18,22-26}$. Six LUCs are investigated in which 8, 64 and 216 atom cells are multiples of Bravais cell, 16, 54 and 128 are multiples of the primitive cells. Table- 1 reference ${ }^{23}$ shows these cells with the expected dimension of the corresponding nanocrystals (after the addition of surface layers). The primitive cell contains two atoms (one boron and one nitrogen atom) while the Bravais cell contains eight atoms (four boron and four nitrogen atoms).

TABLE-1

A COMPARISON BETWEEN THE PRESENT WORK LARGEST INVESTIGATED NANOCRYSTALS (216 ATOM CORE LUC) AND THE BULK EXPERIMENTAL AND CALCULATED PROPERTIES OF CUBIC-BORON NITRIDE OF REFERENCE ${ }^{28}$

\begin{tabular}{lccc}
\hline & $\begin{array}{c}\text { Present 216 } \\
\text { atom cell } \\
\text { properties }\end{array}$ & $\begin{array}{c}\text { Bulk } \\
\text { calculated }^{28}\end{array}$ & $\begin{array}{c}\text { Exp. bulk } \\
\text { properties }^{28}\end{array}$ \\
\hline Lattice constant $(\AA)$ & 3.66 & 3.606 & 3.615 \\
Band gap (eV) & 7.33 & 4.2 & 6.4 \\
Cohesive energy (eV) & 12.9 & 14.3 & 13.2 \\
Valence band width (eV) & 20.6 & 20.3 & $<22$ \\
Ionicity (a.u.) & 0.397 & - & - \\
\hline
\end{tabular}

The surface part is simulated using slab geometry method $^{23-26}$. We chose to simulate hydrogenated (001) Bterminated surfaces of cubic boron nitride since it has the minimum possible reconstruction movements. Most of other surfaces have generally a similar effect on the total electronic structure of a nanocrystal such as reducing the value of the core energy gap.

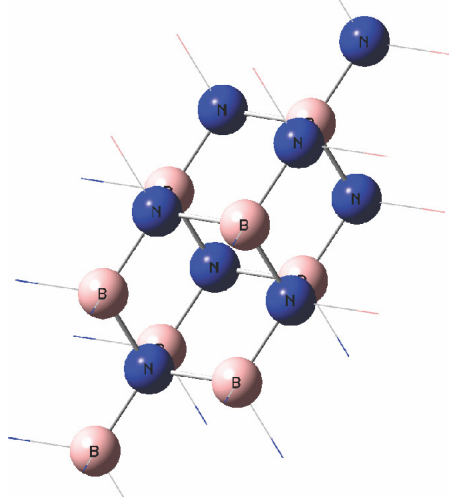

(a)

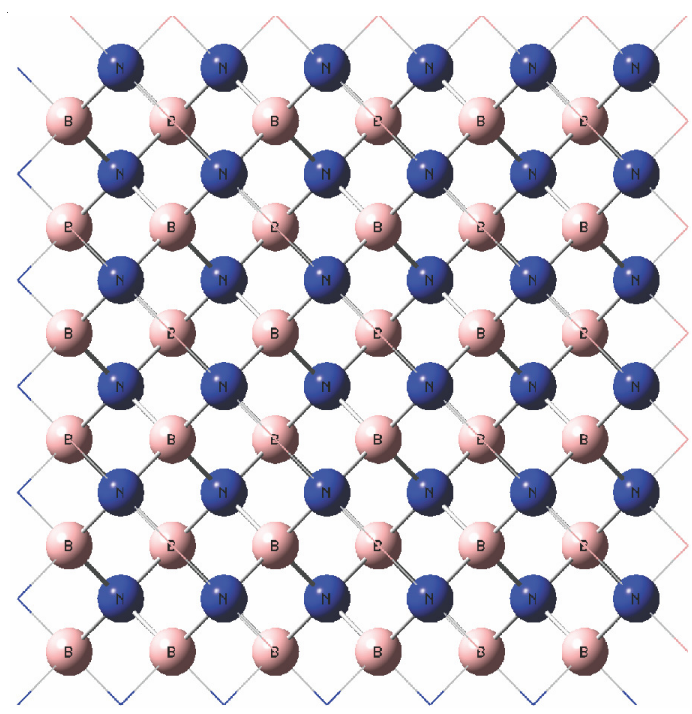

(b)

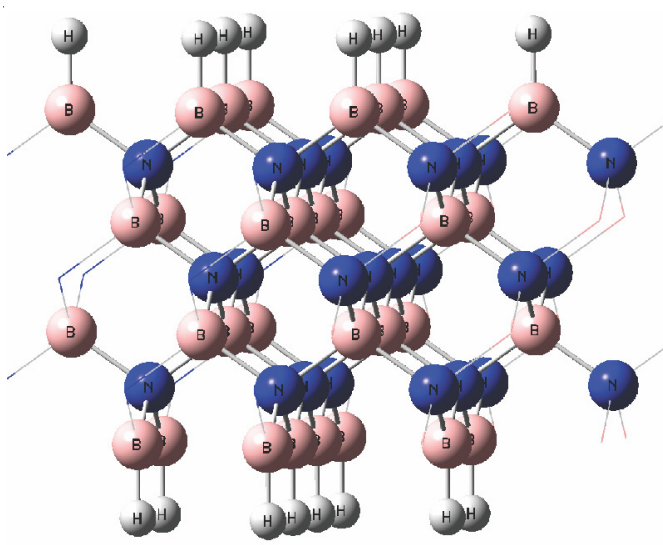

(c)

Fig. 1. (a) LUC of 16 atom core, (b) 216 atom core and (c) hydrogenated boron-terminated $(001)-(1 \times 1)$ surface slab of $4 \mathrm{a}^{2}$ area of boron nitride

Calculations and results: The core part calculations are carried out using 3D periodic boundary condition (PBC) option available in Gaussian 03 program ${ }^{27}$. A central core LUC is formed surrounded by other cells to passivate the outer dangling bonds of the core and to represent the surface effect. The LUCs are energetically minimized with respect to the lattice constant (Fig. 2a). Minimum energy lattice constants 

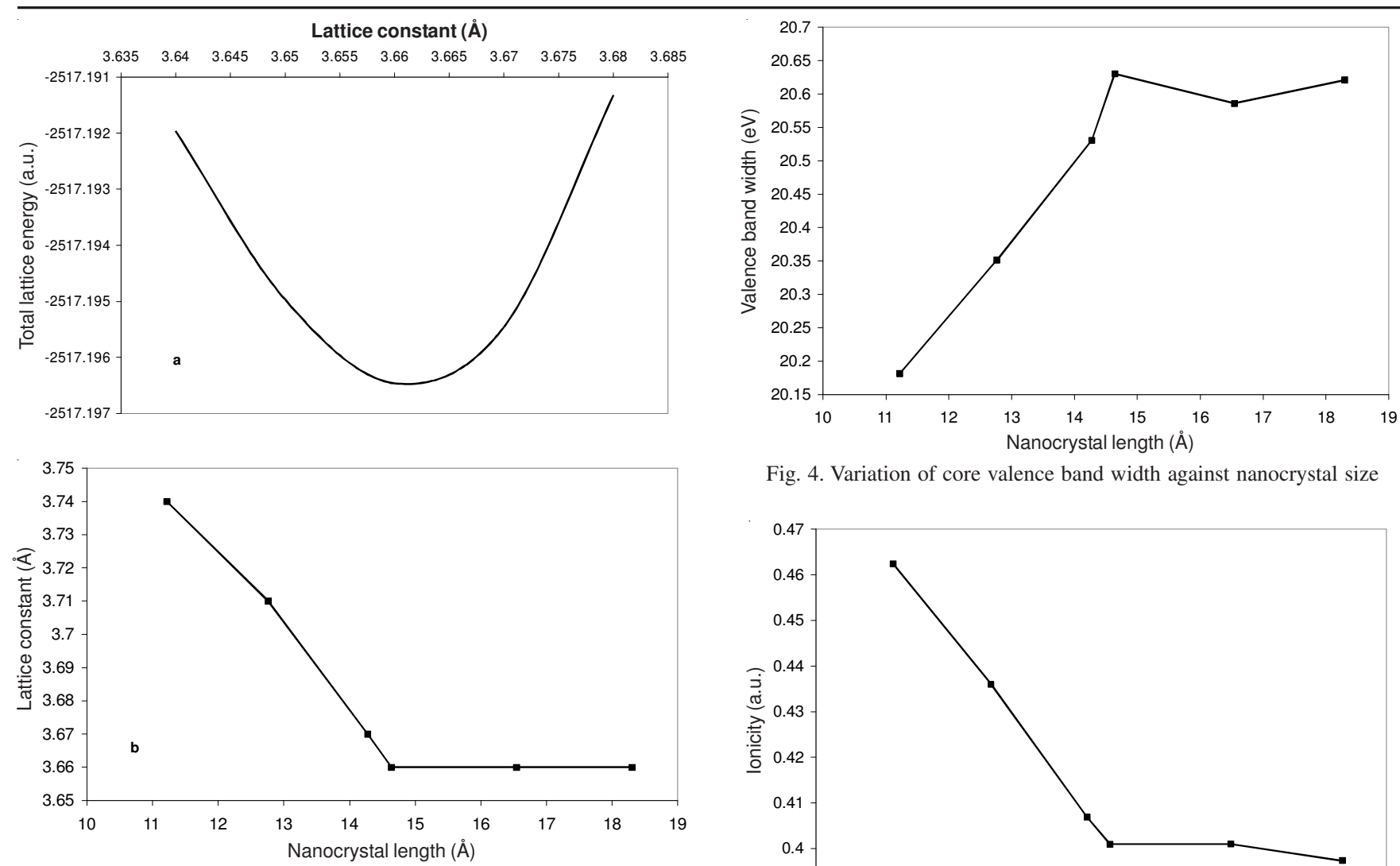

Fig. 2. (a) Total energy of 64 atom core cell against lattice constant and (b) minimum energy core lattice constants against length of nanocrystal

for each cell against the size of nanocrystal are shown in Fig. 2b. STO-3G basis is used with density functional theory coupled with the large unit cell method (LUC-DFT) to simulate the core and surface parts of cubic boron nitride zinc blende nanocrystals. The interaction distance is set to four neighbours which is the width of the surface region. Convergence of the investigated properties can be seen in Figs. 2-6 with some damping oscillations at the convergence region. These figures represent lattice constant, energy gap, valence band width, atomic ionicity and cohesive energy. A comparison between the largest investigated nanocrystals (216 atoms LUC) and the bulk boron nitride crystal experimental and calculated properties of other investigators is given in Table-1.

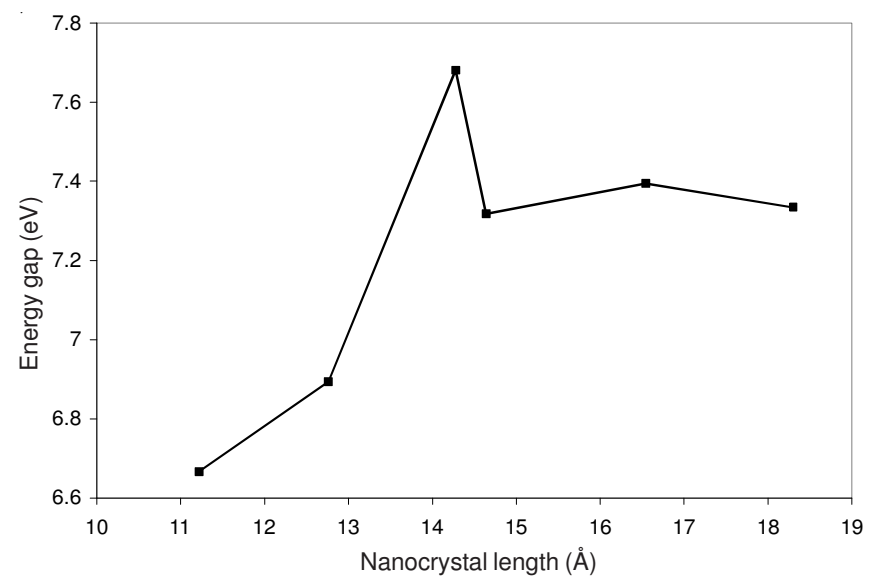

Fig. 3. Variation of energy gap of the core part with nanocluster size

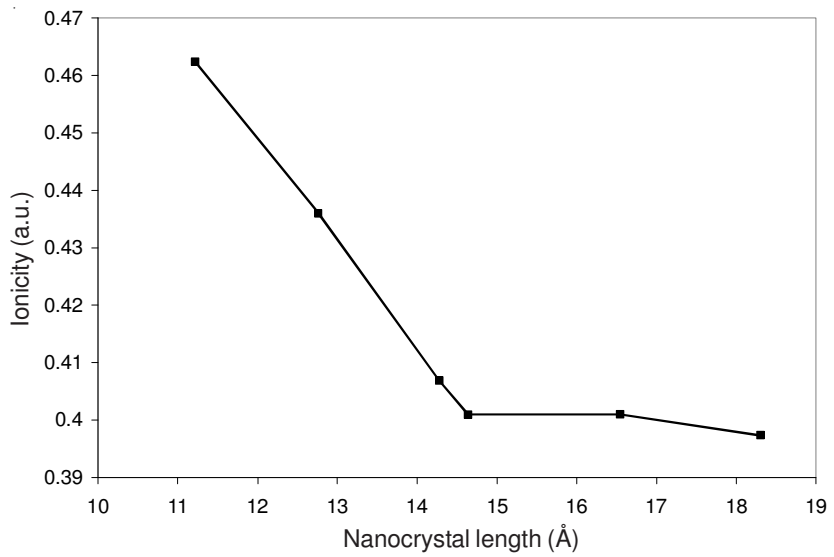

Fig. 5. Atomic ionicity of the core part of different nanocrystal sizes

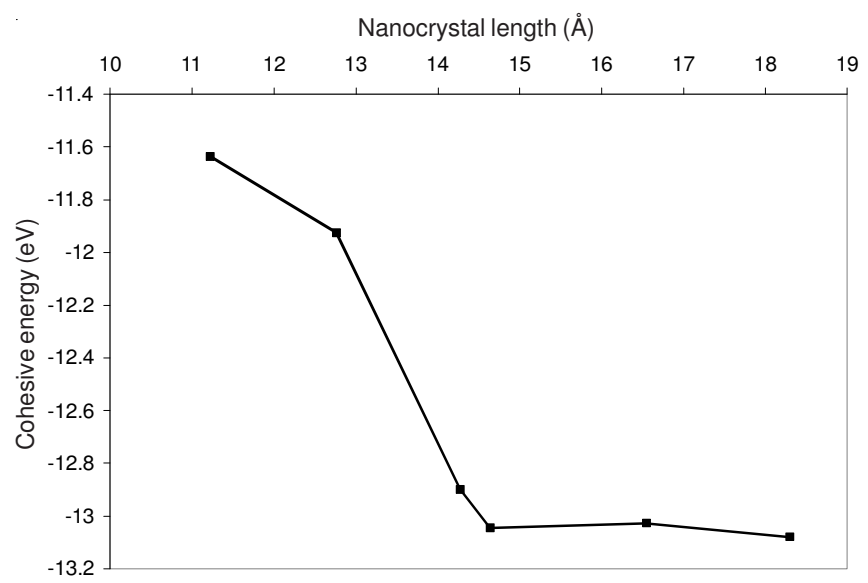

Fig. 6. Variation of core cohesive energy against the size of nanocrystals

Hydrogenated (001) B-terminated surfaces of cubic boron nitride are simulated using slab geometry calculations of $2 \mathrm{D}$ periodic boundary condition available in Gaussian 03 program. Two LUC stoichiometries $\mathrm{B}_{8} \mathrm{H}_{4} \mathrm{~N}_{6}$ and $\mathrm{B}_{32} \mathrm{H}_{16} \mathrm{~N}_{24}$ (Fig. 1c) are investigated. These correspond to two cubic-boron nitride nanocrystals with surfaces $\mathrm{a}^{2}$ and $4 \mathrm{a}^{2}$, respectively, [(a) is the conventional lattice constant]. Larger surfaces are difficult to simulate using the present $a b$ initio method because of long computational time. The resultant degeneracy of states of core 64 atoms LUC and $4 a^{2}$ surface are shown in Fig. 7. Details of the present method can be also found in literature ${ }^{18-21,23-26}$.

Large unit cell method uses partial simulation of a specific part of the nanocrystal that has some kind of symmetry with 

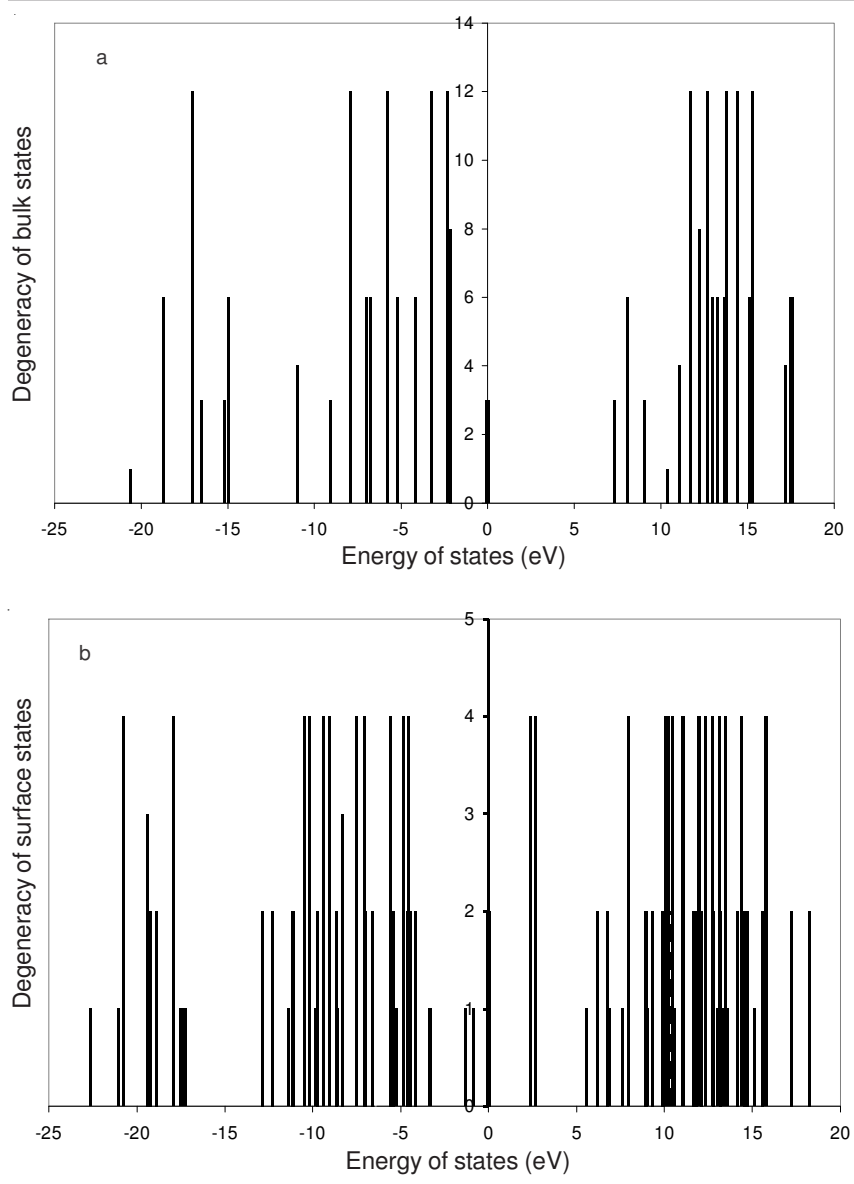

Fig. 7. (a) Degeneracy of 64 atom core, and (b) surface states of LUC surface area $\left(4 \mathrm{a}^{2}\right)$ of hydrogenated B terminated $(001)-(1 \times 1)$ surface. Highest occupied molecular orbital (HOMO) is normalized to 0.0 $\mathrm{eV}$

respect to the dimensions of the nanocrystal. As an example, the core part has a 3D translational symmetry while the surface part has a 2D translational symmetry. In order to facilitate the importance of the method we calculated the electronic structure of a small hydrogenated cubic-boron nitride nanocrystal that has the stoichiometries $\mathrm{B}_{31} \mathrm{~N}_{32} \mathrm{H}_{69}$ (slightly less than $1 \mathrm{~nm}$ length) using the traditional full geometrical optimization with lower level of theory (Hartree-Fock).

\section{RESULTS AND DISCUSSION}

Fig. 2a is a typical molecular or solid state energy curve that relates the total energy of a lattice with the lattice constant. This 64 atom LUC curve is the typical shape of the entire remaining core and surface LUCs energy curves. In Fig. 2b, it is noted that the energetically minimized cells have contracting lattice constant values as the nanocrystals grow up in size. This property was observed experimentally for diamond $d^{29,30}$ and proved theoretically for diamond structured elements using semiempirical and $a b$ initio methods ${ }^{21,23-26}$. However, this is the first theoretical proof and prediction of the existence of such contraction for boron nitride with no experimental data available. This contraction is $2 \%$ of the size of core equilibrium lattice constant of the present largest nanocrystals.

An interesting behaviour of the energy gap of cubicboron nitride nanocrystals core is shown in Fig. 3. The core energy gap fluctuates as nanocrystals grow up in size. The energy gap also shows a damping oscillating behaviour at the convergence region. After a high value at $1.4 \mathrm{~nm}$ (54 atom LUC) the energy gap decreases and increases alternatively. The highest values of the energy gap are associated with the primitive cell multiples $(54,128)$ while the low values are associated with the Bravais lattice multiples $(64,216)$. This shows that not only size but also the shape and symmetry can change the electronic structure of a nanocrystal.

The core valance band width show the same trend of convergence as we go to larger sizes of nanocrystals as shown in Fig. 4. At the converged limit oscillations around the limiting value exist due to different facets and orientations (Bravais or primitive) of nanocrystals as they grow up in size in a similar manner to the energy gap.

The core atomic ionicity of nanocrystal atoms decreases as nanocrystals increase in size and reach slightly below 0.4 a.u. of charge as shown in Fig. 5. This decrease of ionicity has a global effect on the interaction of nanocrystals with their environment and electromagnetic radiation.

Fig. 6 shows the behaviour of the core cohesive energy of boron nitride nanocrystals. The cohesive energy is corrected for zero-point vibration energy which is related to the uncertainly principle ${ }^{21}$. The value of this correction is $0.34 \mathrm{eV}$ per atom pairs ${ }^{28}$. With no exception from the previous energy properties, the cohesive energy decreases in value (increases in absolute value) until it reaches convergence to some limit with very small oscillations around this limit as the nanocrystals grow up in size.

The shapes of the energy gap, valence band width and cohesive energy make us predict that size, symmetry and shape dependence of these quantities oscillate in a way that involves damping oscillation functions that have deep foundations in solid state physics electronic structure ${ }^{22}$. These oscillations are related to the kind of surfaces that are formed as the nanocrystals grow up in size.

Due to its extreme properties, cubic-boron nitride bulk was and is still under heavy investigations $s^{2,28,31-35}$. The largest investigated nanocrystal core in the present work is the 216 atom LUC. This LUC is the nearest to bulk for the present investigated cells. It is customarily to use the biggest size reachable LUC to represent the bulk properties of a crystal ${ }^{18-21}$. As the crystals grow up in size surface effects vanishes and the present core calculations converges to bulk properties. Table-1 summarizes a comparison between reference ${ }^{28}$ bulk experimental and calculated properties with the present core 216 LUC properties. The 216 LUC values are in good agreement with experimental bulk values of boron nitride. Keeping in mind that 216 LUC represents the core of a nanocrystal of 1.8 $\mathrm{nm}$ in size, one can conclude that nanocrystals core properties converge fast to the bulk properties at least for the presently investigated cubic-boron nitride properties. The comparison with other calculation methods in reference ${ }^{28}$ and methods cited in this reference yields an impression of the excellent competition of the present method.

Degeneracy (or density of states if we compare with the bulk) of core and surface states of 64 atom core LUC and hydrogenated B-terminated (001) surface are shown in Fig. 7. This surface is chosen since it is one of the least reconstructed surfaces. Unlike the core part the bond lengths and angles are 
not unique and a variation of geometrical parameters is needed for the surface. Present work optimized B-H bond length is $1.2 \AA$ in good agreement with recent calculations and experiments ${ }^{36}$. The energy gap of the $\mathrm{a}^{2}$ and $4 \mathrm{a}^{2}$ optimized surfaces are 0.48 and $2.37 \mathrm{eV}$, respectively. These values are dramatically less than the core values which show that surface part are the governing part of the energy gap in nanocrystals. The surface reduction of band gap is a universal rule for related diamond structure ${ }^{37}$. The energy gaps have increasing values with nanocrystals size expansion. The same results were obtained for diamond in nearly the same region ${ }^{8}$. This shows that present nanocrystal sizes are outside (beyond) the quantum confinement region. The quantum confinement region imposes an energy gap reduction as nanocrystals grow up in size which is not always the case in the present investigated range.

Fig. 7 also shows that beside the reduction of energy gap both of valence and conduction bands are widened at the surface. The highly degenerate states in the core part are less degenerate at the surface. The reduced symmetry and the introduction of new states that are related to hydrogen atoms at the surface are responsible for the splitting of highly degenerate states in the core part which eventually reduces the energy gap and widens both valence and conduction bands.

The persistent and compact use of symmetry in LUC method leads to a huge reduction of computational time with respect to traditional full geometrical optimization. Computer execution time comparison of the two methods reveals that the small hydrogenated $\mathrm{B}_{31} \mathrm{~N}_{32} \mathrm{H}_{69}$ nanocrystal using the traditional full geometrical optimization takes more than twice the execution time than the largest core of 216 atoms LUC. Knowing that the 216 atom LUC is more than three times the volume of $\mathrm{B}_{31} \mathrm{~N}_{32} \mathrm{H}_{69}$ nanocrystal makes us feel the huge time reduction encountered in LUC method.

As a summary for the present calculations, the lattice constant of cubic-boron nitride nanocrystals is predicted to contract as the nanocrystal grows up in size. This contraction is $2 \%$ of the size of core equilibrium lattice constant for the present size range. The present results show that boron nitride nanocrystals core and surface parts of the presently investigated sizes are outside the well known quantum confinement region that imply a decreasing band gap as the nanocrystals grow up in size. Core energy quantities, such as energy gap, valence band width and cohesive energy all converge to some limit and begin to oscillate with damping at the convergence region. These oscillations are related to the kind of faces that are created as the nanocrystals grow up in size. The good comparison of results between the largest cells and bulk properties reflect the fast convergence of nanocrystal properties to bulk values and the quality of the present method. At the surface, the reduced symmetry and the introduction of new states that are related to hydrogen surface atoms are responsible for the splitting of highly degenerate states at the core part that eventually reduces the energy gap and widens both valence and conduction bands. Compact use of symmetry in LUC method leads to a huge reduction of computational time with respect to traditional full geometrical optimization.

\section{REFERENCES}

1. S. Okada, Phys. Rev. B, 80, 161404(R) (2009).

2. A. Zunger and A.J. Freeman, Phys. Rev. B, 17, 2030 (1978).

3. S.S. Coutinho, V. Lemos and S. Guerini, Phys. Rev. B, 80, 193408 (2009).

4. K. Yuge, Phys. Rev. B, 79, 144109 (2009).

5. J.-C. Zheng, H.-Q. Wang, A.T.S. Wee and C.H.A. Huan, Phys. Rev. B, 66, 092104 (2002).

6. C.X. Wang, P. Liu, H. Cui and G.W. Yang, Appl. Phys. Lett., 87, 201913 (2005).

7. G.W. Yang, Prog. Mater. Sci., 52, 648 (2007).

8. J.Y. Raty, G. Galli, C. Bostedt, Tony W. vanBuuren and L.J. Terminello, Phys. Rev. Lett., 90, 037401 (2003).

9. M. Heidari Saani, M. Kargarian and A. Ranjbar, Phys. Rev. B, 76, 035417 (2007).

10. D.A. Areshkin, O.A. Shenderova, S.P. Adiga and D.W. Brenner, Diamond Rel. Mater., 13, 1826 (2004).

11. N.D. Drummond, A.J. Williamson, R.J. Needs and G. Galli, Phys. Rev. Lett., 95, 096801 (2005).

12. T.M. Willey, C. Bostedt, T. van Buuren, J.E. Dahl, S.G. Liu, R.M.K. Carlson, L.J. Terminello and T. Möller, Phys. Rev. Lett., 95, 113401 (2005).

13. Y.K. Chang, H.H. Hsieh, W.F. Pong, M.-H. Tsai, F.Z. Chien, P.K. Tseng, L.C. Chen, T.Y. Wang, K.H. Chen, D.M. Bhusari, J.R. Yang and S.T. Lin, Phys. Rev. Lett., 82, 5377 (1999).

14. Y.H. Tang, X.T. Zhou, Y.F. Hu, C.S. Lee, S.T. Lee and T.K. Sham, Chem. Phys. Lett., 372, 320 (2003).

15. M. Vörös and A. Gali, Phys. Rev. B, 80, 161411(R) (2009).

16. L. Landt, K. Klunder, J.E. Dahl, R.M.K. Carlson, T. Moller and C. Bostedt, Phys. Rev. Lett., 103, 047402 (2009).

17. G.C. McIntosh, M. Yoon, S. Berber and D. Tomanek, Phys. Rev. B, 70, 045401 (2004).

18. A. Harker and F. Larkins, J. Phys. C, 12, 2497 (1979).

19. M.A. Abdulsattar and K.H. Al-Bayati, Phys. Rev. B, 75, 245201 (2007).

20. I.O. Radi, M.A. Abdulsattar and A.M. Abdul-Lettif, Phys. Status Solidii $B$, 244, 1304 (2007).

21. M.A. Abdulsattar, Physica E, 41, 1679 (2009).

22. C. Kittel, Introduction to Solid State Physics, Wiley, New York, edn. 7 (1996).

23. M.A. Abdulsattar, Solid State Sci., 13, 843 (2011).

24. N.H. Aysa, M.A. Abdulsattar and A.M. Abdul-Lettif, Micro Nano Lett., 6, 137 (2011).

25. N.A. Nama, M.A. Abdulsattar and A.M. Abdul-Lettif, J. Nanomater., 2010, 952172 (2010).

26. M.A. Abdulsattar, Electron. Mater. Lett., 6, 97 (2010).

27. M.J. Frisch, et al., Gaussian 03, Revision B.01, Gaussian, Inc., Pittsburgh PA (2003).

28. R.M. Wentzcovitch, K.J. Chang and M.L. Cohen, Phys. Rev. B, 34, 1071 (1986).

29. O.A. Shenderova and D.M. Gruen, Ultrananocrystalline Diamond: Synthesis, Properties and Applications, William Andrew Inc., edn. 1 (2006).

30. C. Wen, Z.H. Jin, X.X. Liu, X. Li, J.Q. Guan, D.Y. Sun, Y.R. Lin, S.Y. Tang, G. Zhou, J.D. Lin, Guang Pu Xue Yu Guang Pu Fen Xi 5, 681 (2005).

31. D.N. Jayawardane, C.J. Pickard, L.M. Brown and M.C. Payne, Phys. Rev. B, 64, 115107 (2001).

32. F. Aguado and V.G. Baonza, Phys. Rev. B, 73, 024111 (2006).

33. S. Eyhusen, C. Ronning and H. Hofsäss, Phys. Rev. B, 72, 054126 (2005).

34. G. Satta, G. Cappellini, V. Olevano and L. Reining, Phys. Rev. B, 70, 195212 (2004).

35. W. Hehre, L. Radom, P. Schileyer and J. Pople, ab initio Molecular Orbital Theory, Wiley, New York (1986).

36. J. Karlsson and K. Larsson, J. Phys. Chem. C, 114, 3516 (2010).

37. S.J. Sque, R. Jones and P.R. Briddon, Phys. Rev. B, 73, 085313 (2006). 